\title{
Transitions in students' epistemic framing along two axes
}

\author{
Paul W. Irving \\ Department of Physics, Kansas State University, Manhattan, Kansas 66506, USA \\ Mathew Sandy Martinuk \\ Department of Physics and Astronomy, University of British Columbia, Vancouver, British Columbia V6T 1Z1 \\ Eleanor C. Sayre \\ Department of Physics, Kansas State University, Manhattan, Kansas 66506, USA
}

(Received 16 July 2012; published 4 April 2013)

\begin{abstract}
We use epistemological framing to interpret participants' behavior during group problem-solving sessions in an intermediate mechanics course. We are interested in how students frame discussion and in how the groups shift discussion framings. Our analysis includes two framing axes, expansive vs narrow and serious vs silly, which together incorporate and extend prior work on how students frame discussions in physics education research. We present markers for where discussion falls on these axes. We support our conclusions with both microanalytic excerpts of discussion and overall analysis of 75 hours of videobased data. We find that the group spends most of its time in more serious framings, and slightly more than half of its time in more narrow ones. The teaching assistant is the participant who initiates the largest number of frame shifts, and her shifts include bids to all quadrants in the expansive or narrow and serious or silly plane.
\end{abstract}

DOI: 10.1103/PhysRevSTPER.9.010111

PACS numbers: 01.40.Fk

\section{INTRODUCTION}

Previous research on students' epistemic framing in learning contexts has shown that students' framing can influence their choice of learning strategies, their enjoyment of learning activities, and ultimately what is learned [1-3]. Researchers in physics education have demonstrated the benefits of explicitly identifying and attending to students' epistemologies [1,4-6]. Researchers have in the past used analysis of students' discourse, body language, and meta-verbal signals (students' tone of voice, volume of speech, and rhythm of turn taking in conversation) in order to make arguments about students' epistemic framing during collaborative problem solving [7]. These studies are very important because they shed more light on the nature of framing and on the kinds of signals that interacting groups use to communicate with each other to coordinate group activity. Framing is an active bringing together of several resources in an effort to understand and work within a situation [8]. In contrast to framing, epistemological framing is a perception (unconscious or conscious) of what tools and skills are needed in a particular context or situation. Epistemological frames can be envisioned as a storage area for conceptual and procedural resources, promoting some resources' activation and blocking others.

Published by the American Physical Society under the terms of the Creative Commons Attribution 3.0 License. Further distribution of this work must maintain attribution to the author(s) and the published article's title, journal citation, and DOI.
In 2009, Scherr and Hammer published an analysis of students' body language, gaze behavior, and verbal prosody and identified four main clusters of behaviors which were correlated with students' epistemic framing [9]. These clusters corresponded to frames of joking, completing the tutorial worksheet, discussion, and listening to the TA. In their analysis, Scherr and Hammer argue that sensemaking behavior occurs in the discussion frame but they do not distinguish any finer structure of the nature of the epistemic activities that students undertake during discussion. Their paper is an excellent entrée into how groups of students frame discussion in physics, but the theoretical framework has some limitations which we believe can be better captured by a more careful treatment of students' discussion framing, as we will show in this paper.

We are particularly interested in students' changes of framing during discussion: both the factors that trigger a frame shift and the manner of the change. To investigate these dynamics of frame shifts and triggers, we chose to examine students working in a context where there is sufficient evidence to infer students' framing and where there are likely to be many frame shifts. In particular, we examine the context of students engaged in discussion with each other in the presence of a TA during a weekly homework help session (HHS). In this context, students frequently interact with the TA, who responds to questions and tries to guide students to the correct answer on their homework without simply giving out the answers.

The fact that students are primarily engaged in discussion means there will be ample gestural and meta-verbal signals to enable assessment of their framing. The fact that they are engaged in discussion with a TA means that there will be 
ample interactions, both among the students and between the students and TA, that may trigger a frame shift.

After examining our data from the perspective of Scherr and Hammer's categorical coding scheme, we found that we needed some additional theory to describe students' frames before we could talk fruitfully about changes of frames. First, we found that the notion of discrete categories of framings did not fit our data well, and students' conversations instead exhibited a variety of behaviors within one "category," suggesting a more nuanced perspective on the associated framings was needed.

Second, we also noticed students engaging in conceptual discussions even while they were discussing with their TA and while they were joking with each other. Scherr and Hammer's treatment of joking and of TA interactions categorizes them separately from discussions of physics concepts, even in moments where physics concepts are brought into the discussion.

Third, while Scherr and Hammer do identify a discussion frame, they assume that the discussion frame signifies students are engaged in discussion of physics concepts. However, in our data we saw a wide variety in the nature of students' discussions, and noticed some striking differences in the way students were framing the scope of applicability of the topic under discussion. This variation in the narrowness or expansiveness of the discussion echoed earlier work on expansive framing [2] and real-world connections [10], and suggested a need for a look at students' discussion that could distinguish the finer details of the goal and scope of their discussions.

In summary, our data set shows several phenomena that are not well explained by the current theory of students' framing during collaborative discussion:

(1) A wide variation in students' behaviors with subtle, but important differences suggesting that thinking of frames as continua rather than discrete categories is appropriate.

(2) Students engaged in discussion while joking and while interacting with their TA.

(3) Variations in the scope and quality of students' discussion.

In this paper, our goals are the following.

- To further develop theory of how students frame during discussions to account for the three limitations above.

- To illustrate how these developments are supported by our data.

- To account for how students' discussion framing changes.

In the next sections, we briefly introduce our data set before engaging with the goals of the paper; we conclude with a discussion of why this theory is important from both research and practice perspectives.

\section{DATA SOURCES}

The students in this study are enrolled in an intermediate mechanics course and are typically sophomores or juniors. Typically, about 15 students enroll in the course, which is offered annually in the spring semester. Additional details of the course are available in Ref. [8]. An informal weekly HHS is the source of our collaborative problem-solving data. During HHS, students worked in a group to solve homework problems. A graduate student teaching assistant (TA) facilitated the discussion and helped the students toward a solution but did not present problems and their solutions. These sessions were not compulsory in any way, and during the period of data collection, one of the authors (E.C.S.) served as one of the TAs. In a typical HHS, 2-4 students sit around a table with a large table-based whiteboard. Unlike a typical recitation, the TA does not present problems on a chalkboard, and unlike typical tutorials, there is usually only one group of students so the TA stays with them constantly. An unattended video camera records the group from a high angle, capturing gestures as well as their writing on the whiteboard. Additional details about the HHS are available in Ref. [11].

HHS observations were conducted approximately weekly for two iterations of the course, resulting in about 75 hours of video which was composed of 11 different groups. The 11 different groups were often composed of some of the same students but as this was a voluntary session the actual makeup of the groups differed each day of recording. The examples in this paper were chosen after reviewing all of the footage. These clips were chosen as motivating examples and are drawn from several different groups. In all of our analysis, we strongly prefer to use original video because gestural and paraverbal information are important; however, in this paper we present transcribed excerpts with additional notes on tone as appropriate.

\section{THEORETICAL FRAMEWORK}

According to the resources framework [12], students' framing of their activity can have important consequences for how they approach learning situations and the strategies and knowledge they bring to bear [5]. The use of different knowledge in different frames is described in terms of activation of resources.

Resources are myriad small ideas for thinking about physics which can be combined productively to solve problems and make sense of phenomena [13]. Resources are reusable [8] and are connected to each other in networks of mutual activation and/or inhibition [14]. Research using the resources framework focuses on the connections between different ideas in physics $[8,11,13-15]$ and their connections to students' epistemologies [5,16,17]. It has also been applied to other research areas such as epistemic games and frames [18], students' 
sense of physical mechanism [19], student reasoning in novel situations [20], and curriculum development [21-23].

Epistemic frames can be described as a structure of expectations for a person's current activity [9]. A particular frame corresponds to a person's implicit sense of the nature of the activity they are engaging in, and carries with it a network of activations and inhibitions of resources, promoting some resources' activation and blocking others. For instance, in the context of collaborative physics problem solving, a student may activate their resources having to do with classroom physics, but never access their resources related to chemistry or biology. More specifically, in the context of a motion problem in mechanics, a student may activate their resources having to do with equations of motion, energy, Newton's laws, acceleration, and so forth, but never access their resources related to the laws of thermodynamics or Ohm's law. An individual's epistemological framing is highly context dependent, and responsive to both the environment and interactions with other people or artifacts.

A key issue in research on students' framing is the relationship between framing and students' actions such as speech, meta-verbal information, body language, and gesture [24,25]. As described above, framing has to do with the internal state of a student and their currently active set of expectations and associated resources as engaged in an interaction. However, we cannot observe these directly and must instead rely on observations of students' actions. We observe students' actions (and co-occurring clusters of actions) in order to infer what their frame might be, but the actions themselves are not the frame.

Previous researchers have analyzed students' framings in terms of a discrete number of categories [9]. This approach implies that there are discrete categories of framings with sharp boundaries between them. While this may be appropriate for Scherr and Hammer's study which focused on students' behavior, in our study we examine the details of students' speech to interpret their intent [24]. Martinuk, who also conducted analysis on the content of students' speech, used a category-based analysis but describes how the changes between frames occurred over a period of time [26]. This suggests that there is room to augment a category-based analysis of students' framing during discussion.

Instead, we propose a model of epistemic framing in which two axes describe students' framing during discussion. This theory does not attempt to classify all of students' activity during collaborative problem solving, but rather focuses on their framing during discussion with each other. The use of an axis rather than a category is not intended to indicate that we are establishing a formal metric or scale. Rather, we have noticed certain moments in students' conversations may exhibit characteristics that are more or less strongly associated with the extrema of a particular axis, and we want the construct to be able to reflect these differences among students' framings.

\section{A. Expansive and narrow}

Our first axis is narrow framing vs expansive framing. This reflects our desire to highlight when students are focusing exclusively on answering the question at hand vs focusing on discussing more generalizable ideas or exploring the limits of generalizability of an idea. Expansive framing has been discussed by Lam from the perspective of a teacher presenting material as "discuss [ing] an issue that students are likely to be actively engaging with throughout their lives" [27], which Lam places in contrast to "fram[ing] a lesson as a one-time event of learning something that students are unlikely to ever use again."

In observation of students' discussions of physics, we see a similar variation in the implied scope of their discussion. In one instance students may discuss the current physics in conceptual terms that imply its generalizability to other contexts or applications, and in another instance students may discuss the current physics strictly in terms of its relevance to obtaining the answer to the current problem.

To illustrate the differences between expansive and narrow framing, consider the following two examples drawn from HHS.

\section{Example: Expansive}

In this example, a student group is discussing centripetal acceleration in order to understand its meaning.

$T A$ : Centripetal acceleration is it an acceleration or a force?

Chrissy: Force, I think it's a force (looks at Jack), I think.

Jack: Hmm, it's kind of like when you go along, you drive along one of those ramps on the highway and you take a sharp turn and you feel the (shakes body) the pull (shake body one side) like if you turned to the left (motions with one arm to the left).

TA: Uh ha.

Jack: You feel a pull toward (shifts his body to the right) I can't remember if it is away from the direction.

Chrissy: Yeah that is my problem too, is it outward or inward.

Jack: If you're going.

Chrissy: I was so sure it was going inward but I was getting confused.

Jack: (in more definitive tone) If you take a sharp turn you slide the other way (moves whole body to the left).

In the above example, the students demonstrate several behaviors that are associated with expansive framing. For example, we can observe the following.

Real-world connections.-When students cite examples of personal experiences, relevant news items, or real-world applications of the current ideas, it demonstrates that they 
are thinking of the current problem as being connected to things outside the classroom. This shows the expansive scope of ideas that they consider to be relevant to the current situation. In this case, we can clearly see Jack making use of his experiences with driving in a car in order to make sense of the physics of centripetal acceleration.

Gesturing.-Students may use gestures to coordinate multiple representations and connections between their physics classes and the real world. For example, Jack uses gesturing with his hand and his whole body to aid in his visualization of the situation and his communication with Chrissy. He reenacts his prior experiences of what occurred to him when he was in a car turning a corner. Students engaging in a more expansive discussion are more likely to use this type of gesturing to illustrate and communicate their ideas. In general, gesturing may not indicate expansive framing; however, we argue that gesture used to support real-world connections or coordinate multiple representations does indicate expansive framing.

Additionally, in other examples of expansive framing, we notice the following from students.

Use of a variety of representations. - One way in which students display a more expansive approach is by coordinating different representations, such as narrative descriptions of a problem, conceptual representations in terms of physics concepts, mathematical representations (e.g., algebra), and graphical representations. Coordination of formal with informal representations (e.g., algebra and narrative description; algebra and kinesthetics) is considered to be more expansive than coordination of different kinds of formal representations (e.g., algebra and graphs; Cartesian and polar coordinates).

Engage in conceptual discussion.-By its very nature, conceptual information is relevant to more than just the current problem. When students bring up concepts, or when they explicitly talk about the connections between different problems or classes of problems, they demonstrate an expansive framing.

These behaviors are in accordance with previous literature on expansive framing as a mechanism to promote transfer [2] by framing chronologically or representationally $[10,27-33]$ diverse experiences as if they are connected.

We expect that in other data sets, other behaviors might indicate even more expansive framings; however, these behaviors are drawn from our data set only.

The next example illustrates behaviors associated with the narrow end of this axis.

\section{Example: Narrow}

In this example the group is working on a roller coaster problem and are being asked to find all of the forces that are being applied on the cart as it traverses a loop. At the beginning of the transcript they are discussing geometry in order to determine the height of the cart at a certain point on the track.

TA: Yes jump straight to the math part please.

Hugh: Right it is mg times this one (pointing at diagram)

I don't know why it is confusing me so much.

Colin: sine...cosine (indicating both on diagram)

Hugh: Okay.

Group: (long pause)

TA: (directed at Hayley) You don't look convinced.

Hayley: Just trying to understand his drawing.

Alan: This is the theta here so this one is the opposite.

Hayley: Okay.

Alan: This one is the adjacent.

Hayley: Okay that's right.

$T A$ : Okay so do we have an equation for the magnitude of $N$ ?

Hugh: Yeah its that (pointing at an equation on the board).

$T A$ : Okay let's write that down.

In this example students exhibit several behaviors associated with a narrow framing. For example, we observe the following.

Explicit focus on the current problem.-In this example, these students exhibit a focus on "the drawing" and on "the equation," artifacts that are clearly specific to the problem at hand. In other situations, students may demonstrate their focus on the current problem when they reject new ideas or information because they are perceived as irrelevant to answering the problem.

Focus on "what" rather than "why."-When students focus on what to do, they are implicitly working to solve the current problem. Thus, the scope of their framing is reduced to only what will work for the current problem.

Lack of connections to other problems or situations-As above, this indicates that their focus is only on the current problem.

Extended focus on answering of TAs' questions.-This behavior occurs when students are engaged in the narrow framing of "answering the TA's questions." In this behavioral pattern, the TA will ask students questions, but their responses will be quite terse and minimal. After answering, the students will remain silent as though waiting for a TA comment or another question. This pattern may occur both with conceptual questions and with mathematical discussions.

These narrow behaviors are natural opposites to the expansive ones, and reflect students framing their current activities as being very restricted in scope, often focusing only on interpretations of mathematics or physical concepts only in the sense that they are related to obtaining a solution to the current problem.

It is possible, of course, to imagine an expansively framed mathematics discussion in which students use the broad applicability of mathematics to generalize about a broad class of problems, or in which students use varying mathematical and physical arguments to make sense of a 
situation. In this interaction, the students are not using the general applicability of mathematics to frame a problem expansively; instead, they are focusing on the mechanical details of a specific problem (which sides are adjacent and opposite to the angle of interest) in order to solve a tightly defined question (should we use sine or cosine?) so that they can get an equation and write it down. We do not wish to suggest that all uses of mathematics are narrow simply because this example of narrow framing uses mathematics.

\section{B. Categories vs continua}

It is tempting to collapse framings into two categories: narrow framing vs expansive framing. However, this categorical distinction is not supported by our data, whose richness is not supported by this collapse. Instead, we propose that narrow and expansive form two directions on an axis. One interaction in any pair of interactions may be more or less expansive than the other; a collection of interactions may have varying amounts of "expansiveness" as students exhibit expansive behaviors (narrow behaviors) to greater or lesser degrees. In Sec. IV B we introduce the frames plot, which allows for framing to fall along these axes with excellent interrater reliability and can be used to collapse the axes into categories at the expense of losing the pairwise comparisons afforded by the continua.

\section{Serious and silly}

We use a second axis, called the silly or serious axis, to describe moments when students are joking with each other. As described above, the Scherr and Hammer coding scheme collapses instances of on-topic joking along with off-topic discussion and joking. However, in our data we have noticed episodes where students seem to be joking while still being relatively on topic and this on-topic joking can result in a transition to the serious side of the axis. While students' joking may seem to be off topic and unproductive, adopting a "play" framing [25] may allow students to introduce tentative ideas in a way that they can disavow if their peers judge them harshly. We believe this to be one of the main strengths of this framework. That using the two axis framework captures the richness of the data and does not ignore the variation that can occur within a group's use of humor or differentiate between types of discussion.

The silly side of this axis is illustrated by the following example.

\section{Example: Silly}

The students in this example have just finished solving a problem that involved identifying invisible forces affecting an astronaut on a space station who throws a ball and observes its acceleration. Julie begins the example by inquiring what would happen if no ball were dropped.
Although the conversation starts with "real-world" application of a homework problem, it evolves into a discussion of space sickness and then to whether students would like to be astronauts.

Julie: So what if he didn't throw any balls, like, I'm sorry (laughs) what if he didn't drop anything, would he ...(laughs).

TA: This is actually, this is actually a really cool question. Um yes, he does notice, cause your inner ear, as you walk around is incredibly, incredibly sensitive. So because his head is at a sufficiently different radius than his feet, his head feels a different acceleration than his feet do.

Emma: He's going to get sick?

TA: Yeah.

Andy: Yeah, that's what I'm saying.

TA: And he's going to have inner ear imbalance problems?

Emma: I'm going to say that (makes wiggling gesture which evokes physical disequilibration; a couple members of the group laugh).

Julie: Like when you go out into space, sometimes you get sick when you get out there.

TA: That's slightly different.

Julie: Yes it is because you get thrown into ....

Emma: And you feel weightlessness ....

Julie: Yeah.

Emma: All that craziness ....

Julie: Actually, you usually just get sick on the drive up there.

TA: Yeah.

Emma: I wouldn't want to do that.

Julie: I would (all group members laugh).

Julie: Actually, I wouldn't mind being an astronaut if I could.

In this example, the students exhibit the following behaviors that are indicative of silly framing.

Introduce ideas with laughing after an idea.-In the beginning of this example, Julie asks a question that is clearly outside the scope of the homework question and then laughs afterward. This laughter may indicate her nervousness at transgressing the stated goal of the HHS. Regardless, the resulting discussion includes some valuable discussion of the physics of an orbit.

Whole group laughter.-After the students begin discussing whether they would like to be an astronaut there is a moment when the whole group laughs. This clearly communicates that the students are interacting with each other and sharing the same frame, and their laughter expresses that they are more on the silly side of the axis. The laughter at Emma's wiggling movement is friendly, not cruel; the students are not mocking her. Instead, they are sharing her joke.

Although the transcript makes it clear that there is some laughter during this clip, it is not obvious from the transcript whether the students are treating their discussion 
seriously or humorously. Rather, meta-verbal information which we have indicated as the students' tone of voice, volume of speech, and rhythm of turn taking in conversation is important for indicating a silly framing. In the above example, there is a change in the rhythm of the conversation that occurs after the laughter, as well as a shift in the students' gaze behavior from the TA and onto each other. These signals reinforce the assessment that the students have reframed their activity.

In other examples of student interactions in silly framing, we also see students tend to the following.

Comment in a low voice. - as though to communicate only to a single member of the group. This behavior usually indicates a joking comment to a peer.

Use a sarcastic tone of voice.-For assessing this signal of a silly framing, it is especially important to examine the video or audio record, as the transcript alone will not indicate when students are being sarcastic.

Moments of silliness are usually the exception in a collaborative problem-solving session. In that sense, serious framing is more the norm. In fact, the first examples III A 1 and III A 2 in this paper are both serious framing (a feature you probably did not explicitly notice until we pointed it out here).

As students' conversation becomes more serious, they tend to

- Engage in straightforward discussion.

- Pay attention to the TA.

- Pay attention to what the other students are saying and add another on-topic idea to build towards solving the problem.

Students can pay attention to what other students are saying and contribute to what is being said on either side of the silly or serious axis. The distinction between these two framings is the content and intent behind the next idea contributed. While framing a discussion as silly the humorous aspects of an idea will be built upon. Conversely while framing a discussion as serious, an idea that builds towards solving the problem will be introduced. Because we observed moments when students were joking while being on topic as well as moments when students are joking off topic, we believe the axislike nature of this construct is justified. We also observe both joking and seriousness in all aspects of the narrow or expansive axis. Therefore, we believe the two axes are at least somewhat independent.

In the following sections, we apply this theory to examine a large data set and discuss several phenomena that pertain to frame transitions.

\section{ACCOUNTING FOR FRAME SHIFTING}

Our third goal in this paper is to account for students' frame shifts in discussions. How does a group maintain a frame? What prompts members to change discussion frames? How long do frames last? In what ways is the TA a special agent for frame change?
During HHS, students generally remained in a given frame for as little as 10 seconds to beyond 5 minutes. Shorter duration frames were often silly episodes sandwiched between longer serious frames. A frame could shift within one statement from an individual but this statement would have to be acknowledged by the rest of the group in order for a frame transition to occur. Transitions between HHS sessions often began in the silly and narrow quadrant as students joined the group and settled in before reading the first problem. During this time, students engaged in gossip or conversation about activities in the last week. When the group starts a problem, the discussion becomes more serious. The group spends the majority of the time in serious discussion ranging all over the narrow or expansive axis, occasionally becoming more silly for brief jokes or funny comments until the problem is solved. After each problem, the group enters a nondiscussion period to write down solutions, which may then develop into a silly and narrow off-topic discussion until work on a new problem starts.

\section{A. Frame changes}

In the following example, the group moves from a somewhat expansive and silly framing to one which is more narrow and serious.

\section{Example: Changing frames}

Just prior to this example, the group reads a problem about the forces in rotational motion. They have difficulties visualizing the problem, and they realize that seeing a rotating body will help.

$T A$ : We are looking for things to spin to see it better. (A secondary TA is looking for an item to spin. There are a few jokes about the first effort they put forth, which is far too big. Then the TA attaches some string to a roll of tape.)

Chrissy: Ah its nice.

TA: Okay get this thing going...make it spin.

Group: (laughs)

Chrissy: (picks up the tape on string and swings it) Swing in a vertical radius...okay you try (to Jack).

Jack: Like that (swings it) okay I'm going to put this down before I kill somebody.

TA: Yeah.

Janet: (picks it up and swings better than previous two) Chrissy: Oh you're good.

TA: Do you practice?

Group: (laughs)

Jack: Well I majored in.

TA: (pause) OK so what's going on in the problem?

Chrissy: So we have, I'm going to draw a diagram, we don't have air resistance (drawing on her notepad) so we have mg...(continues explanation of her diagram).

To focus on the framing changes in this interaction, we need to slightly abstract the groups' actions. The episode starts when the group decides to coordinate different representations of the motion (physical object moving in a 
circle and kinesthetic sense of forces with verbal problem statement), an indication of expansiveness. Initially, they frame this coordination seriously.

Their search for an appropriate object to twirl turns to joking. The TA, Chrissy, Jack, and Janet take turns swinging it and commenting on their abilities. This activitymanipulating actual objects-is not common in HHS, and the group becomes quite silly. The silliness is exemplified in the Chrissy and the TA's commentary on Janet's swinging. The paraverbal information is important here: the group (and we) interpret their statements as friendly banter rather than serious inquiries about Janet's past twirling experience.

After all students swing the tape, the TA bids to return to the task at hand: the worksheet problem they are solving. Her tone is serious, and her attention turns from the swinging tape to the table in front of them. The rest of the group also attends to the problem in front of them, narrowing their focus and becoming more serious.

\section{B. Frame prevalence}

To chart the relative seriousness or silliness and expansivity or narrowness of students' framings in longer interactions, we developed the "frames plot" (Fig. 1).

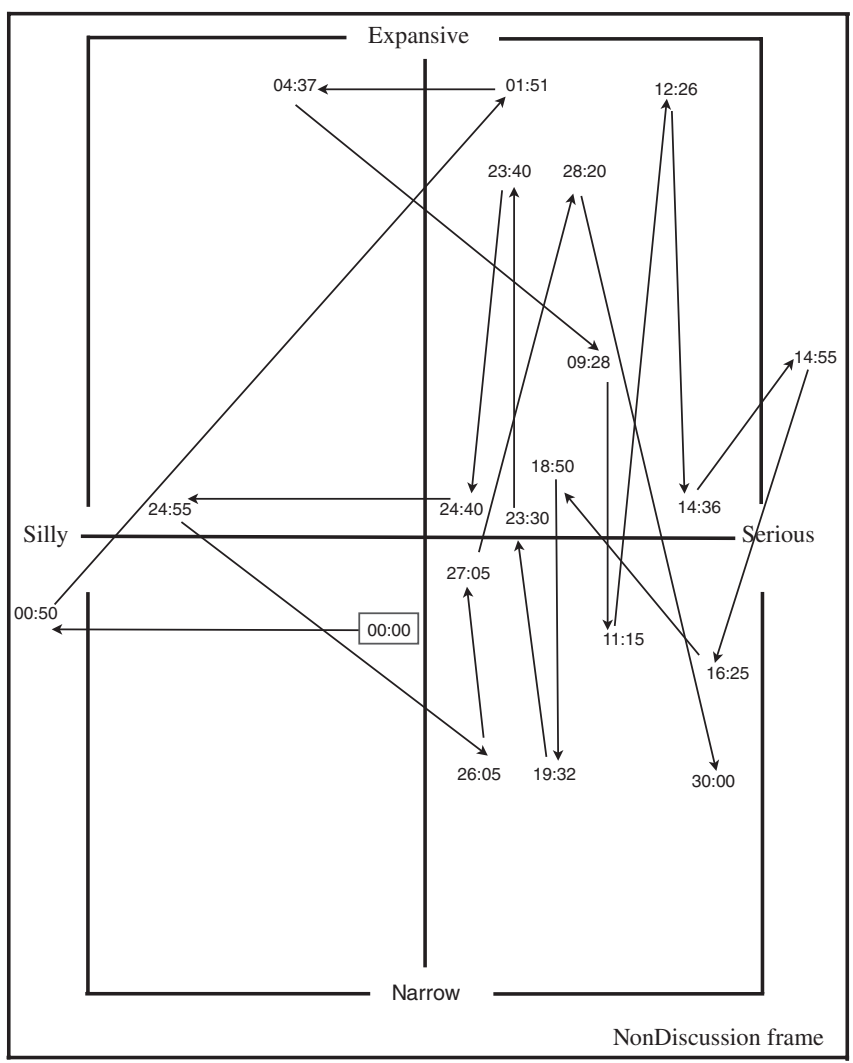

FIG. 1. The frames plot. Expansive or narrow is on the vertical axis and serious or silly is on the horizontal axis. The outer box delineates discussion framing (in the box) from nondiscussion framing (out of the box). Time stamps in each location indicate the time the group entered the frame. The data in this plot are a representative sample of 30 minutes of HHS.
The choice of what clusters of behaviors fall near each axis on the plot is normed to our sense of what usually happens when students discuss physics. Different normings are possible-we expect that what constitutes "serious" in a war room differs significantly from what constitutes serious in a comedy club-and the important feature is comparing changes of framing with time, not the value of each framing. That said, to give a holistic sense of the data, it is useful to collapse the continuous axes into four discrete categories (the quadrants on the frames plot), noting that categorical labels do not capture the variation in the interactions well. A transition is not only the movement from one quadrant to another. Obviously, transitions between quadrants are much more obvious. As was stated above the plot is normed to our sense of what usually happens when students discuss physics and so transitions within quadrants were often recognized. For example, when students' conceptual discussion began to incorporate more representations and made a direct application to the real world but were engaged in a conceptual discussion prior to this, this would be considered a within serious and expansive quadrant transition. Figure 2 represents the fraction of the total HHS time that students spent in each quadrant and the nondiscussing frame.

To test the frames plot and the validity of the coding scheme, five researchers independently coded the same video clip after brief discussion of the nature of the plot. After coding, the researchers met again to compare their plots and discuss normalization. While some differences in degree of silliness or seriousness and expansiveness or narrowness existed between researchers, there were very few differences in quadrants or in number of frame changes before the renormalization discussion. After renormalization, all researchers agreed on the appropriate plot. A single researcher coded all remaining video. On several occasions during the final analysis process, additional researchers would "check in" on the data analysis by reviewing completed frames plots together with

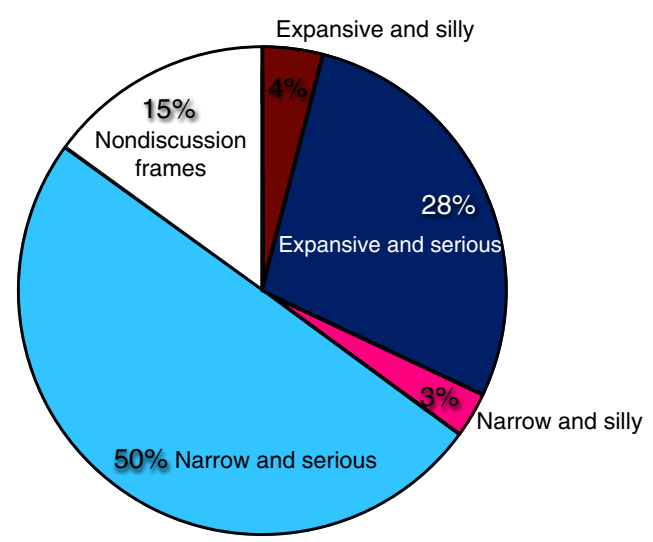

FIG. 2 (color online). Each slice denotes the fraction of time in each quadrant. 
attendant video, and checking for agreement with the original researcher's analysis. This process produced excellent agreement between the researchers for the results presented below. The same process was used to analyze the tactics that caused a shift in frame and who initiated the transition data.

In total 56 hours of HHS learning environment data was analyzed using the frames plot with $1 \%$ equating to 33 minutes. Interactions tend to be more expansive 32\% of the time, and more narrow $53 \%$ of the time. An additional $15 \%$ of the video does not fall onto this chart because the axes are meant only for discussion, and students do not discuss anything during that time (Fig. 2). For the most part, during nondiscussion time, the students are writing on their worksheets instead of talking.

In our data set, we notice that, overall, students do not engage in the behaviors that indicate silliness very often, nor do sillier framings last for very long. The majority of the time that is spent on the silly side of the axis occurred at the beginning of the HHS session or at the end of the solution of the problem before the recording of the answer begins. Occasional jokes, inferences, or miscommunications during the session resulted in the students transitioning onto the silly side. If they occurred in the middle of the session, these sojourns into silliness were often very quick and the group or the TA would quickly reframe and return to more serious discussion.

\section{THE TA AS A SPECIAL PARTICIPANT}

In our data set and in contrast to previous research on TAs in groups (see, e.g., Refs. [9,34]), the TA's constant presence does not mean that the TA interrupts the normal work of the group: she is a member of the group already, and we conceptualize her contributions as if she were a participant. However, the TA is a special participant. The group acknowledges that she knows more physics than the other members and that her contributions are meant to guide them in the "right" direction. However, the group does not expect that the TA will tell them the answers nor perform solutions (though she is expected to help them when they are stuck), nor do they expect her to grade their work or see the problem statements before the HHS. The TA has a dual purpose in the HHS learning environment: to aid students in the construction of a solution to specific homework problems, and to foster the development of their understanding. These two purposes prompt the TA to alternately narrow and expand, respectively, the group's framing. Changes to the group's framing are frequently initiated by the TA; occasionally the TA will bid to change the framing and the group does not change.

We identified the type of behavior the TA engaged in before the group transitioned from one quadrant to another in order to clarify the relationship between TA interventions. For example, in example the TA initiates the move to be more serious and narrow at the end. In example the TA

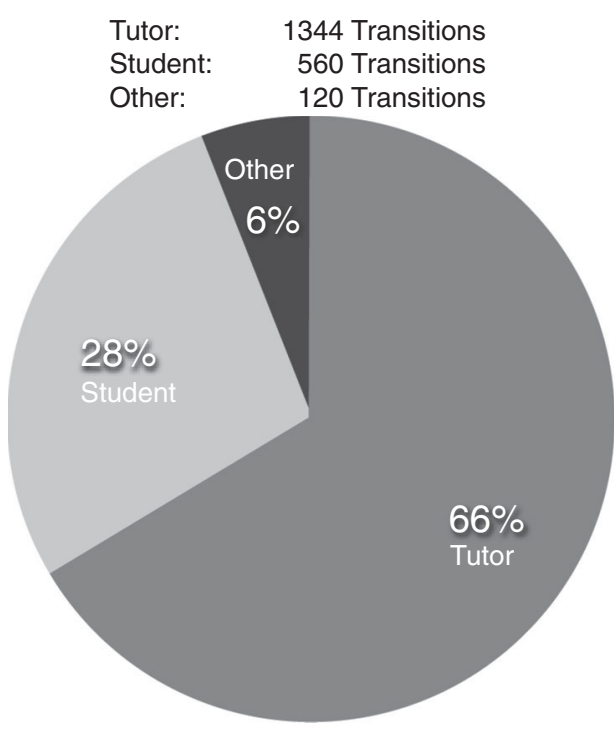

FIG. 3. Who initiates a transition to another frame.

picks up on Julie's expansive cue and introduces the astronaut's inner ear, prompting the group into a more silly framing. The majority of time the TA transitions the group from one quadrant of the axis to another (Fig. 3). These data were collected during the frames plot analysis and are based on the same 56 hours of video recordings. In regard to Fig. 3 other represents times when a transition in frame occurred without the TA or students verbally instigating it such as an occurrence external to the group or a prolonged silence. Although the silence would end with either a tutor or student saying something, it was the silence itself that would result in the transition.

Figure 4 is a breakdown of the types of transitions TAs used to encourage students from quadrant to quadrant

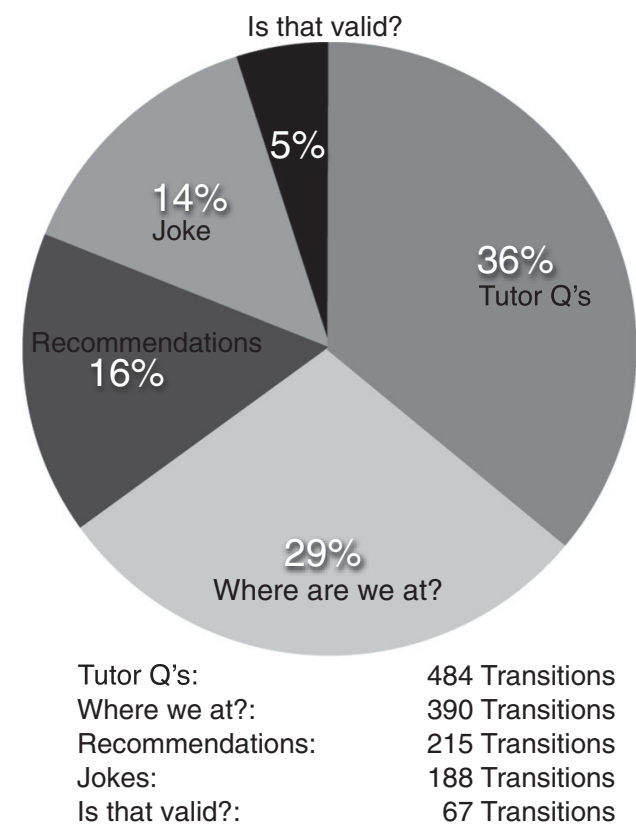

FIG. 4. Moves that TAs use to transition to another frame. 
obtained from the same 56 hours of video that was analyzed using the frames plot. It must be noted again at this stage that although the TA can attempt to instigate a reframing of the interaction it is up to the students to interpret and concur with this attempt at reinterpretation. From the results it is clear that the majority of the time it was simply the TA asking a question. Both "is that valid?" and "where are we at?" have been excluded from this category as they figured so prominently as transitions in the learning environment. "Where are we at?" was a question the TA would ask regularly in order either to focus the students on the goal of the problem or to start the HHS session, i.e., which problem do you want to work on (see the last line in example: changing frames). This could result in a transition to either serious and narrow or serious and expansive. "Is that valid?" often came up at the end of the solution to a problem or if an idea that warranted more discussion was presented. It would typically result in a transition to serious and expansive but not exclusively. In regard to other questions, a very high proportion of the serious and expansive interactions were instigated simply by the TA questioning the students as to why they had done something. A combination of a TA consistently questioning a student's explanation while not giving the students their own explanation seems to encourage the students to frame the instance as serious and expansive. TAs used a variety of questions to achieve this, such as Why? How do we know that is true? Are you sure? Does everyone understand? or as evidenced in the expansive example "Is it an acceleration or a force?" This method, called "reflective toss" [35], "throws" the responsibility for elaboration back to the student. It is an attempt to help students clarify meaning, consider a variety of views, and monitor their own thinking. The other typical transitions are "jokes" which can be implemented by both the TA and the students (see example silly) and "recommendations" which is when the TA asks the group to try something (see example narrow-the first line).

\section{DISCUSSION}

\section{A. Limitations in applicability}

A significant difference between the circumstances of this study and others' circumstances is that the TA is always present with the groups. We chose this environment because we were especially interested in framing changes, and we suspected that the TA's presence might prompt many changes. Our suspicions were correct.

The results of this study also indicate that the TA has a significant influence on how students epistemologically frame their learning environment and that TAs are often the instigators of transitions from one frame to another [1]. We note that the TA can be a major influence on the framing of an activity by students in this learning environment, but they are not the only frame tipper nor are all their bids to tip the frame accepted. The students have to both interpret and be willing to follow the cues and tipping that the TA is encouraging. This was not always the case in this learning environment with the difference being that the goal of the students partaking in the HHS learning environment was probably a determining factor.

Given that the TA influences the epistemic frames of the group, one might question why the TA would encourage a silly frame to be adopted in the learning environment. One possibility is that TAs who allow transitions into silliness allow students to be more comfortable expressing risky ideas. [It is also possible that the TAs encourage silliness (and the students participate in it) because it makes the HHS more fun for them. These two goals are not mutually exclusive.] There is a need for further research to examine the role that play takes in a collaborative learning environment [36]. We postulate that joking is necessary in a collaborative learning environment in order for all the other frames to successfully occur.

Furthermore, the dual intent of the HHS-broadening students' understanding of physics while doing specific homework problems in a group-requires both narrow and expansive framings. However, we have already normalized what counts as "narrow" and what counts as "expansive" to the kinds of framings we see in this situation. We expect that other situations may encourage different expansive framings which focus on bringing together chronologically distant ideas [2,27] instead of different representations.

\section{B. Implications for practice}

This paper has several possible implications for informing practice in small group learning environments. The examination of factors that trigger frame shifts and which factors result in which type of transition especially with the focus on TA prompted transitions gives an insight into how expansive framing can be encouraged in group learning environments. Expansive framing is a mechanism for promoting transfer [2,37] and relating diverse experiences together in a learning environment and should therefore be encouraged by instructors [27]. We also highlight that on-topic discussion does occur while groups are framing an activity as expansive silliness and that the TA has a role to play in encouraging framing an activity as silly. Instructors should not automatically view a laughing group as one that is off topic: students may be having fun in a relevant way.

\section{Usefulness to researchers}

The two axes framework for analyzing group discussion suggests many future research questions. It enables a finer examination of the nature of the epistemic activities and the intricacies of the discussion of students engaged in group learning environments. The fact that only $15 \%$ of the data analyzed did not fit into the framework indicates how appropriate this type of analysis is for group learning environment data. Also incorporating the joking frame and the TA interactions into the two axis analysis of the 
discussion captures the richness of the data that would have been lost with earlier analyses [9]. Further study of humor in learning groups is warranted, as are comparative analyses of different learning environments. Though our data are taken from a specific learning environment, we envisage our framework as transferable to other group learning environments which focus on discussion as a main part of the learning activity.
[1] P. Hutchison and D. Hammer, Attending to student epistemological framing in a science classroom, Sci. Educ. 94, 506 (2010).

[2] R. A. Engle, Framing interactions to foster generative learning: A situative explanation of transfer in a community of learners classroom, J. Learn. Sci. 15, 451 (2006).

[3] R. Duschl, Science education in three-part harmony: Balancing conceptual, epistemic, and social learning goals, Rev. Res. Educ. 32, 268 (2008).

[4] L. Lising and A. Elby, The impact of epistemology on learning: A case study from introductory physics, Am. J. Phys. 73, 372 (2005).

[5] D. Hammer and A. Elby, Tapping epistemological resources for learning physics, J. Learn. Sci. 12, 53 (2003).

[6] D. Hammer, E.F. Redish, A. Elby, and R. E. Scherr, Resources, framing, and transfer, in Transfer of Learning: Research and Perspectives, edited by J. Mestre (Information Age Publishing, Charlotte, NC, 2004).

[7] B. W. Frank and R. E. Scherr, Interactional processes for stabilizing conceptual coherences in physics, Phys. Rev. ST Phys. Educ. Res. 8, 020101 (2012).

[8] E. C. Sayre and M. C. Wittmann, Plasticity of intermediate mechanics students' coordinate system choice, Phys. Rev. ST Phys. Educ. Res. 4, 20105 (2008).

[9] R.E. Scherr and D. Hammer, Student behavior and epistemological framing: Examples from collaborative active-learning activities in physics, Cognit. Instr. 27, 147 (2009).

[10] E. Forman and E. Ansell, Orchestrating the multiple voices and inscriptions of a mathematics classroom, J. Learn. Sci. 11, 251 (2002).

[11] E. C. Sayre, M. C. Wittmann, and J. E. Donovan, Resource plasticity: Detailing a common chain of reasoning with damped harmonic motion, in Physics Education Research Conference 2007, edited by L. McCullough [AIP Conf. Proc. 883, 85 (2007)].

[12] A. A. diSessa, Toward an epistemology of physics, Cognit. Instr. 10, 105 (1993).

[13] D. Hammer, Student resources for learning introductory physics, Am. J. Phys. 68, S52 (2000).

[14] M. C. Wittmann, Using resource graphs to represent conceptual change, Phys. Rev. ST Phys. Educ. Res. 2, 020105 (2006).

[15] K.E. Black and M.C. Wittmann, Epistemic games in integration: Modeling resource choice, in Proceedings of the 2007 Physics Education Research Conference, edited by L. Hsu, L. McCullough, and P. R. Heron [AIP Conf. Proc. 951, 53 (2007)].
[16] A. Gupta, A. Elby, and C. Park, Beyond epistemological deficits: Incorporating flexible epistemological views into fine-grained cognitive dynamics, in Proceedings of the 2010 International Conference of the Learning Sciences, Chicago, 2010 (International Society of the Learning Sciences, Chicago, 2010), Vol. 2, pp. 372-373.

[17] A. Gupta, D. Hammer, and E. Redish, The case for dynamic models of learners' ontologies in physics, J. Learn. Sci. 19, 285 (2010).

[18] J. Tuminaro and E.F. Redish, Elements of a cognitive model of physics problem solving: Epistemic games, Phys. Rev. ST Phys. Educ. Res. 3, 020101 (2007).

[19] R. S. Russ, V. R. Lee, and B. L. Sherin, Framing in cognitive clinical interviews about intuitive science knowledge: Dynamic student understandings of the discourse interaction, Sci. Educ. 96, 573 (2012).

[20] E. C. Sayre, M. C. Wittmann, and J. R. Thompson, Resource selection in nearly-novel situations, in Physics Education Research Conference Proceedings 2003, edited by K.C. Cummings, S. Franklin, and J. Marx (Springer, New York, 2003).

[21] A. Elby, E. F. Redish, and D. Hammer, "Helping students learn how to learn: Open-source physics worksheets integrated with TA development resources," National Science Foundation proposal, Project No. 0341447, University of Maryland, 2004-2007 (unpublished).

[22] B.S. Ambrose, Investigating student understanding in intermediate mechanics: Identifying the need for a tutorial approach to instruction, Am. J. Phys. 72, 453 (2004).

[23] T. I. Smith and M. C. Wittmann, Comparing three methods for teaching Newton's third law, Phys. Rev. ST Phys. Educ. Res. 3, 020105 (2007).

[24] D. Tannen, Framing in Discourse (Oxford University Press, New York, 1993).

[25] G. Bateson, A Theory of Play and Fantasy (Ballantine, New York, 1972).

[26] M. Martinuk, Epistemological Framing and Sensemaking in Collaborative Group Problem-Solving in Introductory Physics, doctoral dissertation, University of British Columbia, 2012 (unpublished).

[27] D. P. Lam, X. Meyer, R. A. Engle, L. Goldwasser, S. Perez, K. Zheng, J. Clark, E. Naves, H. Rosas, and D. Tan, A microgenetic analysis of how expansive framing led to transfer with one struggling student, in Proceedings of the Biennial Meeting of the International Conference of Learning Sciences, Sydney, 2012 (International Society of the Learning Sciences, Sydney, 2012).

[28] S. Ainsworth, P. Bibby, and D. Wood, Examining the effects of different multiple representational systems in 
learning primary mathematics, J. Learn. Sci. 11, 25 (2002).

[29] M. J. Lawson and M. Chinnappan, Knowledge Connectedness in Geometry Problem Solving, Knowl. Creat. Diffus. Util. 31, 26 (2008).

[30] P. Thompson and J. Silverman, The concept of accumulation in calculus, in Making the Connection: Research and Teaching in Undergraduate Mathematics, edited by M. Carlson and C. Rasmussen (Mathematical Association of America, Oberlin, OH, 2007), pp. 117-131.

[31] J.F. Wagner, C.a. Manogue, J.R. Thompson, N.S. Rebello, P. V. Engelhardt, and C. Singh, Representation issues: Using mathematics in upper-division physics, AIP Conf. Proc. 1413, 89 (2012).

[32] A. Witherspoon, R. Azevedo, and G. Lewis, Adolescents' use of multiple representations of information in selfregulated and externally-regulated learning with hypermedia, in Proceedings of the 30th Annual Conference of the Cognitive Science Society, edited by B. Love, K. McRae, and V. Sloutsky (Cognitive Science Society, Austin, TX, 2008), pp. 2359-2364.
[33] R. A. Engle, S. Roberts, P. D. Nguyen, P. Yee, and Framing Transfer Research Group, A design-based approach to experimental design: Investigating hypotheses about how framing influences transfer, in Proceedings of the International Conference of the Learning Sciences, Utrecht, Netherlands, 2008 (unpublished).

[34] R. M. Goertzen, R.E. Scherr, and A. Elby, Accounting for tutorial teaching assistants' buy-in to reform instruction, Phys. Rev. ST Phys. Educ. Res. 5, 020109 (2009).

[35] E.v. Zee, Using questioning to guide student thinking, J. Learn. Sci. 6, 227 (1997).

[36] L. Conlin, A. Gupta, and D. Hammer, Where to find the mind: Identifying the scale of cognitive dynamics, in Proceedings of the 2010 International Conference of the Learning Sciences, Chicago, 2010 (International Society of the Learning Sciences, Chicago, 2010), Vol. 1, pp. 277-284.

[37] R. Engle, The resurgence of research into transfer: An introduction to the final articles of the transfer strand, J. Learn. Sci. 21, 347 (2012). 